\title{
A new optimization approach for indoor location based on Differential Evolution
}

\author{
A. D. Masegosa ${ }^{1,2}$ A. Bahillo ${ }^{1}$ E. Onieva ${ }^{1}$ P. Lopez-Garcia ${ }^{1}$ A. Perallos ${ }^{1}$ \\ ${ }^{1}$ Deusto Institute of Technology, University of Deusto, 48007, Bilbao, Spain \\ ${ }^{2}$ IKERBASQUE, Basque Foundation for Science, 48011, Bilbao, Spain
}

\begin{abstract}
The growth of Location Based Services and Location Aware Services in indoor environments has focused the attention of the research community on indoor location systems, especially on those based on WLAN networks and Received Signal Strength (RSS). Despite the advances reached, the development of reliable, accurate and low-cost indoor location systems still remains as an open problem. In this work, we focus on a specific class of location methods where the position of a Mobile Station (MS) is estimated by optimizing a cost function. As far as we know, the optimization models for indoor location proposed so far, only consider the current RSS measurements to estimate the position. In this paper, we propose an optimization approach that uses both current and past measurements to estimate the MS location. To solve the underlying optimization problem we use a Differential Evolution algorithm. The experimentation done over a simulated and a real scenario shows, on the one hand, that using past and current measurements we obtain more accurate and robust position estimations, and on the other hand, that our proposal is competitive versus other high-performing location methods proposed in the literature.
\end{abstract}

Keywords: Indoor Location, WLAN, RSS, Evolutionary Algorithms, Differential Evolution

\section{Introduction}

Indoor location systems have received increasing attention recently due to their important applications in areas as healthcare, safety, logistics, etc. Although there have been many advances, the development of robust, accurate and cheap indoor location systems is still an open problem.

The great availability and low cost of WLAN IEEE 802.11 wireless networks have made them one of the most popular technologies for this purpose. The objective of these wireless location systems consists on estimating the position of a Mobile Station (MS) from some measurements taken from the signals provided by Access Points (APs). The location process is usually divided in two steps: 1) acquisition of location metrics from APs; and 2) processing of location metrics to estimate MS position. These measurements can be Time-Of-Arrival (TOA), Time-Difference-OfArrival (TDOA), Received-Signal-Strength (RSS), or Angle-Of-Arrival (AOA) [1]. Among all of these metrics, RSS presents the best accessibility and availability, especially in 802.11 networks where the RSS indicator (RSSI) can be easily obtained.

Due to the usual complex and changing conditions of the propagation environments of these signals, it is very difficult to predict or model the variation of the RSSI values. To address this problem, one of the most common approaches proposed in the literature consists on modelling the variation of the RSS measurements by means of a previous calibration phase to obtain a statistical model from which we can obtain location methods based on fingerprinting [2]. The main drawbacks of these methods are the cost of the calibration phase (the accuracy of the model depends on the amount of measurements taken [3]), and its sensibility to changes in the environment (obstacles, devices, people moving, furniture changes, etc.) [4], which usually make these methods inappropriate for dynamic environments.

In order to solve these limitations, researchers have proposed different approaches that do not need a calibration phase. Some proposals are based on modifications of the wireless network, for example, by incorporating external devices that dynamically estimate the propagation model [5]. There are also location methods that do not require the modification of the network. These methods can be classified in three classes [6]: Geometric-based methods, which depart from estimations of the distances between the MS and the APs, and determine the position by solving algebraic equations that relates these distances with the MS location;Bayesian methods, that estimate the position of the MS from a conditional probability distribution that predict the most probable position given all the past RSS measurements; and Optimization-based methods, where the estimation of the position is done by finding a location that minimizes a cost function.

In this work, we have focused on the third class of methods. The objective function to optimize in this type of approaches is usually based on the so called path-loss model [7] that relates RSS measurements and distances to the corresponding AP. These approaches estimate the MS position by finding the solution that minimizes the Maximum Likelihood formulation for this model [8]. This problem has shown to be very complex mainly because of its 
high multimodality and noise. To avoid this problem, some authors has proposed the used of more detailed models that include information about the environment (e.g. presence of walls between MS and AP) [9] or by imposing some constraints that limit the space of feasible solutions[10].

As far as we know, the optimization models for indoor location proposed so far, only consider the current RSS measurements to estimate the MS location in contrast to other approaches, as Bayesian methods, that make use of current and past measurements for the estimation process. By using past measures we can provide more information to the model which may lead to more accurate estimations. The main drawback that adding more information may entail is a bigger complexity of the objective function. In this sense, Evolutionary Algorithms [11] can be a powerful tool to deal with the resulting optimization problem, given their success in complex problems of other fields [12].

With these ideas in mind, we propose a new optimization-based approach for indoor location. Concretely, the model presented here estimates the MS position from the RSS measurements taken at the current instant $t$ and at the instant $t-1$. To solve the underlying optimization problem, we used a Differential Evolution (DE) algorithm [13].

To test our strategy we use a simulated and a real scenario. The experimentation done over these scenarios aims, on the one hand, at analyzing the benefits of using past and current RSS measurements versus using only the current ones, and on the other hand, at checking the competitiveness of our proposal with respect to other reference indoor location methods proposed in the literature.

The paper is structured as follows. The next section gives basic definitions and concepts about RSS indoor location based on the path-loss model. Our proposal is presented in Section 3, where we describe the cost function designed to estimate the MS position, and the DE algorithm developed to solve it. Section 4 is devoted to show the experimental framework, describing the scenarios used to test our proposal and the implementation details. The results of the experimentation are analysed in Section 5. Finally, we discuss the main conclusions of the paper in Section 6 .

\section{Indoor location based on path-loss model}

As said before, optimization methods for indoor location based on RSS measurements usually estimate the MS position employing the so called pathloss model[7], an empirical model that relates signal strength and distance to the corresponding AP. According to this model [7], the signal strength received from an AP is given by:

$$
P_{R}=P_{0}-10 \gamma \log _{10}\left(\frac{d}{d_{0}}\right)+X
$$

where $P_{R}$ is the RSS value for the signal when the distance between AP and MS is $d$, whereas $P_{0}$ is the RSS measured at a reference distance $d_{0}$. $P_{0}$ can be known in advance [7], by a simple calibration process, and its value will remain the same as long as the antenna gains and the transmitted power do not change. Constant $\gamma$ is called the path-loss exponent, and determines the velocity at which the attenuation experienced by the signal increases because of the distance between the wireless nodes [14]. This parameter depends on the propagation environment of the signal and the higher its value, the higher the attenuation at a given distance. $X \sim \mathbb{N}(0, \sigma)$ represents the log-normal shadowing effect [15].

Assuming we have $N$ APs and $d_{0}=1$, from Equation 2, we can formulate the Maximum Likelihood Estimation (MLE) of the MS position as [8]:

$$
\begin{aligned}
\min _{\boldsymbol{x}} H(\boldsymbol{x})=\sum_{i=1}^{N} \frac{1}{\sigma_{i}^{2}} & {\left[\left(P_{0}-P_{R_{i}}\right)\right.} \\
& \left.-10 \gamma_{i} \log _{10}\left(\left|\boldsymbol{x}_{i}-\boldsymbol{x}\right|\right)\right]^{2}
\end{aligned}
$$

where $P_{R_{i}}, \boldsymbol{x}_{i}$ and $\gamma_{i}$ are the RSS value received from the $i-t h \mathrm{AP}$, the location of the $i-t h \mathrm{AP}$ and the corresponding path-loss exponent, respectively. Since many factors (obstacles, devices, people, etc.) can influence the propagation environment, assuming path-loss exponents as known is an oversimplification of the reality. To avoid this, path-loss exponents can also be estimated. In this case, the joint MLE of the MS location can be formulated as:

$$
\min _{\theta} H(\theta)=\sum_{i=1}^{N} \frac{1}{\sigma_{i}^{2}}\left[\left(P_{0}-P_{R_{i}}\right)-10 \gamma_{i} \log _{10}\left(d_{i}\right)\right]^{2}
$$

where $\theta=\left(\boldsymbol{x}, \gamma_{1}, \ldots, \gamma_{N}\right)$ and $d_{i}=\left|\boldsymbol{x}_{i}-\boldsymbol{x}\right|$. As mentioned above, the resolution of this problem results in a non-convex highly multi-modal optimization problem with a high number of local optima. To reduce the complexity of this cost function, many authors have proposed the use of constraints based on connectivity information (estimated distance to the AP) and geometry in order to reduce space of feasible solutions and/or the number of local optima. In this work, we will take the heuristic constraints proposed in [10] and [16] given their good results. Assuming, without loss of generality, that the order of the $N$ APs according to their RSS values is $A P_{1}, A P_{2}, \ldots, A P_{N}$, these heuristics constraints are defined as follows:

1. The path-loss exponents lie between a maximum and a minimum value, that is, $\gamma_{\min } \leq$ $\gamma_{i} \leq \gamma_{\max }$

2. Since the deployment of APs in the network is usually homogeneous, we can assume that the distance from MS to the AP with the strongest RSS value, $d_{1}$, should be lower than a constant $D 1$. This constant is chosen as the furthest distance from any possible MS position to the nearest AP. 
3. If the signal strength received from $A P_{i}$ is similar to that of $A P_{1}$, for $i \neq 1$, we can assume that $A P_{i}$ is also at a distance lower than $D 1$ from the MS position. In this way, if $P_{R_{1}}-P_{R_{i}}<C 1 \Rightarrow d_{i} \leq D_{1}$.

4. From the definition of $D 1$, we can delimitate the distance between MS and the other APs by $D 1$ and the distance between $A P_{1}$ and the other APs in the following way:

$$
\begin{aligned}
\mid \operatorname{dist}\left(A P_{1}, A P_{i}\right) & -D 1 \mid \leq d_{i} \\
& \leq \operatorname{dist}\left(A P_{1}, A P_{i}\right)+D 1
\end{aligned}
$$

where $\operatorname{dist}(x, y)$ is the Euclidean distance between the points $\boldsymbol{x}$ and $\boldsymbol{y}$. The interested reader may refer to [10] for a graphical explanation of this heuristic constraint.

5. Although $P_{R_{i}}>P_{R_{j}}$ does not necessarily imply that $d_{i}<d_{j}$, when the difference between both RSS values is big enough, we can assume that $A P_{i}$ is closer than $A P_{j}$. Therefore, we assume that if $P_{R_{i}}-P_{R_{j}}>C 2 \Rightarrow d_{i}<d_{j}$.

\section{Description of the new optimization approach for indoor location}

As we mentioned in the introduction, the main motivation behind our proposal is the use of past and current RSS measurements to provide more accurate estimations of the MS position. With this idea in mind, and assuming that there is a IEEE 802.11 wireless network deployed in a environment, the objective of our approach is to track, every $T_{s}$ time units, the position of a MS that is moving within this environment. To this end, we make the following assumptions:

- The MS can measure RSS values from all APs in range, and $M$ measures are taken during a time interval of $T_{M}$ time units. $T_{M}$ is taken in such a way that we can assume that the environment and the distance to all APs does not change significantly.

- The interval of time between position estimations, $T_{s}$, is equal or greater than the time spent to take the $M$ measurements, that is, $T_{s} \geq T_{M}$.

- $P_{0}$ is known and remains constant as long as there are no modifications in antenna gains and transmitted power. As said before, $P_{0}$ can be easily obtained $[17,7]$.

- The path-loss exponents $n_{i}$ are unknown and can vary from one instant to the other, due to obstacles or changes in the environment, which is the usual situation in real scenarios.

- The path-loss exponents remain unchanged during the time interval $T_{M}$. When the time period $T_{M}$ is short enough, the path-loss exponents fulfil this condition [18].

- The distance travelled by the MS in $T_{s}$ time units is lower than a certain threshold $D_{\max }$. If $T_{s}$ is relatively short, this distance can be easily delimited. For example, if the MS is carried by a person walking in a building and $T_{s}$ is set to 1 second, we can set $D_{\max }$ to a value in the range $[0.9,1.3]$ meters, since the usual velocity in this case varies from 3 to $4.5 \mathrm{Km} / \mathrm{h}$.

Having said that, the proposal presented in this paper estimates the MS position at the current instant $t_{c}$ from the measurements taken in this instant and in the former instant $t_{f}$, where $t_{c}-t_{f}=T_{s}$. We will refer as $\overline{P_{R}^{t_{c}}}$ and $\overline{P_{R}^{t_{f}}}$ to the mean of the $M$ RSS measurements taken during the time intervals $\left[t_{c}-T_{M}, t_{c}\right]$ and $\left[t_{f}-T_{M}, t_{f}\right]$, respectively. The next part of the paper is devoted to describe the objective function proposed to estimate the MS position from current and past sensor measurements and the DE algorithm designed to find the solutions that minimize this function.

\subsection{Description of the objective function}

We refer to the MS position at the instant $t$ as $\boldsymbol{x}^{\boldsymbol{t}}$. Generally speaking, the cost function described here estimates the current MS location $x^{t_{c}}$ by finding those positions $\boldsymbol{x}^{t_{c}}$ and $\boldsymbol{x}^{\boldsymbol{t}_{\boldsymbol{f}}}$ that minimize their respective Maximum Likelihood formulations, given $\overline{P_{R}^{t_{c}}}$ and $\overline{P_{R}^{t_{f}}}$, and whose separation distance $\left(\left|\boldsymbol{x}^{\boldsymbol{t}_{c}}-\boldsymbol{x}^{\boldsymbol{t}_{\boldsymbol{f}}}\right|\right)$ is lower than $D_{\max }$. More formally, the objective function $G$ that estimates the position $x^{t_{c}}$ is defined as:

$$
G\left(\theta^{t_{c}}, \theta^{t_{f}}\right)=H\left(\theta^{t_{c}}\right)+H\left(\theta^{t_{f}}\right)
$$

where $\theta^{t_{c}}=\left(\boldsymbol{x}^{t_{c}}, \gamma_{1}^{t_{c}}, \ldots, \gamma_{N}^{t_{c}}\right)$ and $\theta^{t_{f}}=$ $\left(\boldsymbol{x}^{t_{f}}, \gamma_{1}^{t_{f}}, \ldots, \gamma_{N}^{t_{f}}\right)$. The function $H\left(\theta^{t}\right)$ corresponds to the Maximum Likelihood formulation for the MS location at the instant $t$ and is defined as:

$$
H\left(\theta^{t}\right)=\sum_{i=1}^{N} \frac{1}{\left(\sigma_{i}^{t}\right)^{2}}\left[\left(P_{0}-\overline{P_{R_{i}}^{t}}\right)-10 \gamma_{i}^{t} \log _{10}\left(d_{i}^{t}\right)\right]^{2}
$$

$G$ is subject to the following constraints:

$$
\begin{aligned}
\gamma_{\min } \leq \gamma_{i}^{t} & \leq \gamma_{\max } \\
d_{1}^{t} & \leq D_{1} \\
\text { if } \overline{P_{R_{1}}^{t}}-\overline{P_{R_{i}}^{t}}<C 1 & \Rightarrow d_{i}^{t} \leq D_{1} \\
\left|\operatorname{dist}\left(A P_{1}^{t}, A P_{i}^{t}\right)-D 1\right| & \leq d_{i}^{t} \\
& \leq \operatorname{dist}\left(A P_{1}^{t}, A P_{i}^{t}\right)+D 1 \\
\text { if } \overline{P_{R_{i}}^{t}}-\overline{P_{R_{j}}^{t}}>C 2 & \Rightarrow d_{i}^{t} \leq d_{j}^{t} \\
\operatorname{dist}\left(\boldsymbol{x}^{t_{c}}, \boldsymbol{x}^{t_{f}}\right) & \leq D_{\max }
\end{aligned}
$$

where $t \in\left\{t_{c}, t_{f}\right\}, i=1 \ldots N$ and $j=1 \ldots N$. Equations (7) to (11) corresponds to the heuristics constrains described in Section 2. Equation (12) limits the maximum distance between $\boldsymbol{x}^{t_{c}}$ and $\boldsymbol{x}^{\boldsymbol{t}_{\boldsymbol{f}}}$ to $D_{\max }$. In order to avoid the model dealing with feasible and unfeasible solutions, we use a penalty function $P$ whose value is proportional to the number of constraints violated, that 
1 Initialize $P=\left\{i n d_{1}, \ldots\right.$, ind $\left._{P_{\text {size }}}\right\}$ randomly;

2 gens $=0$, gensLastRestart $=0$;

3 while gens $<\operatorname{maxGenerations~do~}$

4 for $\left(i=1\right.$ to $\left.P_{\text {size }}\right)$ do

$5 \quad / *$ Mutation step */

$6 \quad$ Randomly select two individuals $i n d_{r 1}$ and ind $_{r 2}, r 1 \neq r 2$

ind $^{\prime}=$ ind $_{i}+F \cdot\left(\right.$ ind $_{\text {best }}-$ ind $\left._{i}\right)$ $+F \cdot\left(i n d_{r 1}-i n d_{r 2}\right)$;

/* Crossover step

ind $d^{\prime \prime}=$ binomial_crossover $\left(\right.$ ind $_{i}$, ind $\left.^{\prime}\right)$;

/* Local search step

Apply local search method to $i n d^{\prime \prime}$;

/* Replacement step

if $\left(g\left(i n d^{\prime \prime}\right)<g\left(i n d_{i}\right)\right)$ then

ind $_{i}=$ ind $d^{\prime \prime}$

if $\left(g\left(i n d^{\prime \prime}\right)<g\left(\right.\right.$ ind $\left.\left._{\text {best }}\right)\right)$ then

$\mid i n d_{b e s t}=i n d^{\prime \prime}$

end

end

end

gens $=$ gens +1

gensLastRestart $=$ gensLastRestart +1 ;

/* If the performance obtained so far */

/* is bad, the search is restarted */

if $\left(g\left(\right.\right.$ ind $\left.\left._{\text {best }}\right)>\rho\right) A N D$

(gensLastRestart $>$ maxGenerations $/ 3)$ ) then

Re-initialize population $P$ randomly; gensLastRestart $=0$;

end

if $\left(g\left(\right.\right.$ ind $\left.\left._{\text {best }}\right)<\mu\right)$ then

break;

end

33 end

34 return ind $_{\text {best }}$

Algorithm 1: Differential Evolution Pseudo-code

is, $P\left(\theta^{t_{c}}, \theta^{t_{f}}\right)=N V C \cdot \rho$, where $N V C$ is the number of constraints violated and $\rho$ is a penalty factor. In this way, the objective function to optimize is $G\left(\theta^{t_{c}}, \theta^{t_{f}}\right)=H\left(\theta^{t_{c}}\right)+H\left(\theta^{t_{f}}\right)+P\left(\theta^{t_{c}}, \theta^{t_{f}}\right)$.

\subsection{Differential Evolution Algorithm}

In this subsection we will describe the method designed to solve the optimization problem resulting from our proposal. Since the high multimodality of the MLE model is inherited by our proposal, we decided to use a DE algorithm [19] to solve it, given its good performance in continuous multimodal problems [13]. To be more specific, we have used a Memetic DE in order to improve the exploitation capabilities of this method. Each individual ind corresponds to a solution and it is encoded as: $i n d=\left(\theta^{t_{c}}, \theta^{t_{f}}\right)$. For the sake of the simplicity, the fitness of an individual ind $=\left(\theta^{t_{c}}, \theta^{t_{f}}\right)$ is given by $g($ ind $)=G\left(\theta^{t_{c}}, \theta^{t_{f}}\right)$. The pseudo-code of the method can be seen in Algorithm 1.

As we can see in the pseudo-code, lines 1 to 20 correspond to a common DE/current-to-best/1 algorithm [13] with binomial crossover [13] and cou-

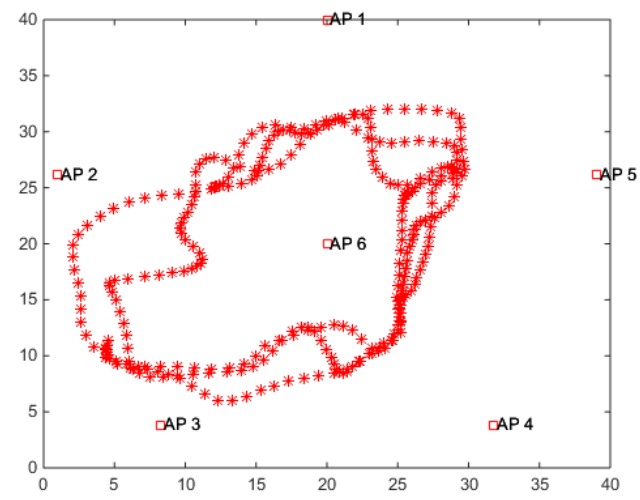

Figure 1: Simulated scenario $\left(40 \times 40 \mathrm{~m}^{2}\right)$ with 6 anchors placed on the vertices and center of a regular pentagon. Red points correspond to the trajectory followed by the MS.

pled with a local search. Lines 23-27 aims at restarting the search if the method has not found a feasible solution $\left(g\left(\right.\right.$ ind $\left.\left._{\text {best }}\right)>\rho\right)$ in a reasonable amount of time (maxGenerations/3). Finally, the objective of the condition 'if' in line 28 consists on stopping the search once we have found a good solution, in order to return a position estimation as soon as possible.

\section{Experimental Framework}

This section is devoted to describe the two scenarios used to test our proposal and the implementation details of our approach.

\subsection{Simulated scenario}

The simulated scenario can be seen in Figure 1. This scenario consists on a $40 m \times 40 m$ square with 6 APs placed in the vertices and center of a regular pentagon with a $20 \mathrm{~m}$ radius. The trajectory of the MS was generated by simulating a person walking with at a maximum velocity of $5 \mathrm{~km} / \mathrm{h}$ between two concentric circles center in $(20,20)$ and with $5 \mathrm{~m}$ and $18 \mathrm{~m}$ radius, respectively. A total of 305 points were generated. For each point in the trajectory, the $M$ RSS values corresponding to the time interval $\left[t-T_{M}, t\right]$ were generated according to Equation 2, where $M$ was set to 100 . In the latter equation, $P_{0}$ was set to $-56.5 \mathrm{dBm}$, and the $\gamma_{i}$ were simulated as uniform random variables. In particular, $\gamma_{1} \in U(1.3,1.7) ; \gamma_{2}, \gamma_{3} \in U(1.7,2.25)$; $\gamma_{4}, \gamma_{5} \in U(2.25,3.25)$, and $\gamma_{6} \in U(3.25,4.25)$, where $\left(\gamma_{1}, \gamma_{2}, \ldots, \gamma_{6}\right)$ are the 6 different path-loss exponents that characterize the propagation channel from the 6 APs sorted according to their proximity to the MS. Finally, the standard deviation of the shadow fading $X$ was simulated as a uniform random value between $2.85 \mathrm{dBm}$ and $3.45 \mathrm{dBm}$.

\subsection{Real scenario}

The second scenario corresponds to real measurements performed at the second floor of the Higher 
Technical School of Telecommunications (ETSIT) at the University of Valladolid in Spain. As shown in Figure 2, the campaign of measurements was carried out following a route among offices, laboratories and with a few people walking around. A total of 123 points were considered in the route. As anchors, 8 identical wireless access points (AP) were used with two omnidirectional rubber duck antennas vertically polarized to each other in diversity mode. The APs were configured to send a beacon frame each $100 \mathrm{~ms}$ at constant power on frequency channel $1(2.412 \mathrm{Ghz})$. As MS, an IEEE 802.11b WLAN cardbus adapter was used with two on-board patch antennas in diversity mode. Diversity circuitry determines which antenna has better reception and switches it on in a fraction of a second while it turns off the other antenna. Therefore, both antennas are never on at the same time. Both APs and cardbus adapter can be found on most IEEE 802.11 WLANs. For the location process, we only considered the four most powerful APs.

\subsection{Implementation details}

To finish with the description of the experimental framework, we will give some details of the implementation and the parameter settings used for the experimentation.

Regarding the objective function, according to the guidelines given in [16] and [10], the constants $\mathrm{C} 1$ and $\mathrm{C} 2$ were set to $6 \mathrm{dBm}$ for both scenarios. D1 was set to 10 and 17 meters for the simulated and the real scenario, respectively, whereas $P_{0}$ was assigned to -56.5006 and 52 , in the same order. As for the $D_{\max }$ parameter, its value was fixed to 2 and 1 meter for the simulated and the real scenario, respectively, according to the maximum velocity of the MS in each case. Finally, the penalty factor $\rho$ was set to 0.5 based on previous experimentation.

The parameters of the DE algorithm were established according to standard values and previous experimentations. Concretely, the population size, the parameter $\mathrm{F}$ and the crossover probability were set to 50, 0.8 and 0.5, respectively. We used Hill Climbing as local search operator. The maximum number of generations was 500 and 400 for the simulated and the real scenario, in that order. The parameter $\mu$ that establishes when a good estimation of the position has been reached was set to 0.01 .

All models and algorithms were implemented in Java 8, and the experiments were run in a computer with Windows 7 Operative System, Intel Core i54210 and 4G RAM. All the source codes and data are available upon request.

\section{Result Analysis}

The experimentation done has two objectives:

- Assessing the benefits of using both current and past RSS measurements versus using only the

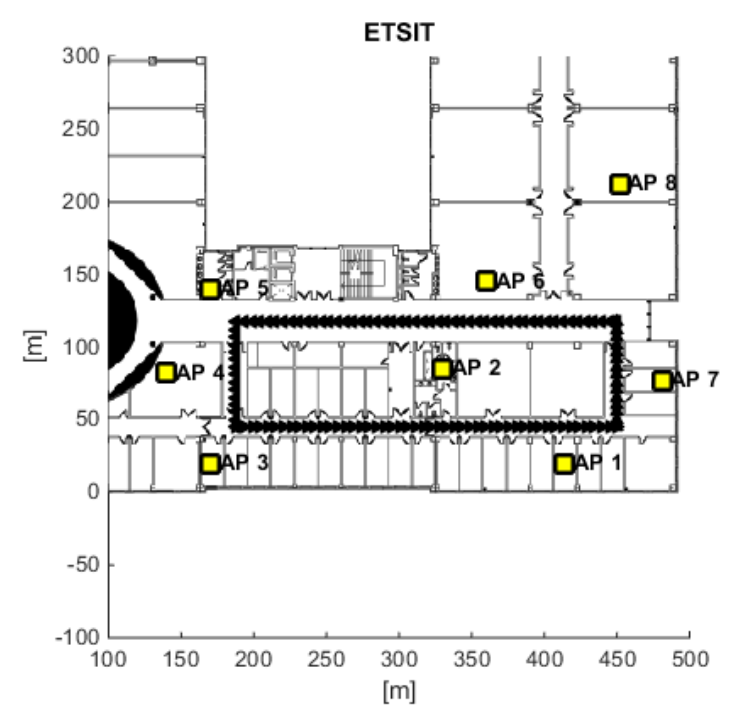

Figure 2: Real scenario corresponding to RSS measurements taken in the second floor of the Higher Technical School of Telecommunications of the University of Valladolid in Spain. Triangular points correspond to the trajectory followed by the MS.

current one, which is the common approach in optimization methods for indoor location.

- Comparing the performance of our proposal versus other reference methods in literature.

The remaining part of this section is devoted to analyze the results of this experimentation.

\subsection{Analyzing the benefits of using current and past RSS measurements}

As mentioned above, the first objective of the experimentation was to evaluate whether using past and current RSS measurements leads to more accurate estimations or not. To this end, we considered two different approaches:

- Current-RSS-DE: This approach estimates the MS position by the objective function given in Equation 6 and subject to constraints from 7 to 11 . It only uses the current RSS measurements $\overline{P_{R}^{t_{c}}}$.

- Current+Past-RSS-DE: The second approach estimates the position by the objective function given in Equation 5 subject to constraints from 7 to 12 . It uses both the $\underline{\mathrm{RSS}}$ measurements taken at the current instant $\overline{P_{R}^{t_{c}}}$ and in the previous one $\overline{P_{R}^{t_{f}}}$.

In both cases, the DE algorithm described in Section 3.2 was used to find the positions and path-loss exponents that minimize the corresponding objective function. Since DE is a stochastic method, we run the algorithm 15 times for each point in the trajectory of the MS. Given that the trajectory of the simulated scenario consists of 305 points and the real one of 123 , we have a total of $15 \times 305=4575$ 


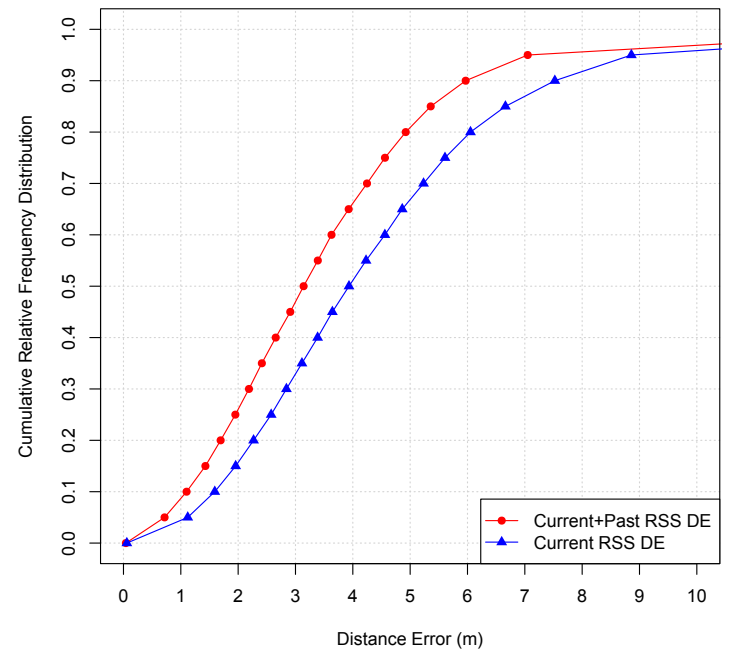

Figure 3: Cumulative Relative Frequency Distribution (CRFD) for the distance errors (in meters) of the estimations of Current-RRS-DE (blue-triangle line) and Current+Past-RSS-DE (red-circle line) in the simulated scenario.

and $15 \times 123=1845$ location estimations, respectively. To determine the accuracy of these estimations we have employed the Euclidean distance between the estimated position and the real position.

Figures 3 and 4 display the Cumulative Relative Frequency Distribution (CRFD) for the distance errors (in meters) of the estimations for both approaches in the simulated and the real scenario, in that order. Focusing on the simulated scenario, Current+Past-RSS-DE (red-circle line) outperforms Current-RSS-DE (blue-triangle line) since the distance error of its estimations are much lower along the whole distribution. Furthermore, the differences in accuracy grow for higher percentiles. For example, the differences in distance error for the percentiles 25, 50, 75 and 95 are $0.63 \mathrm{~m}, 0.79 \mathrm{~m}$, $1.05 \mathrm{~m}$ and $1.81 \mathrm{~m}$, respectively. This shows the bigger robustness of the Current+Past-RSS-DE approach since, in this scenario, it obtains an average (median) distance error of $3.14 \mathrm{~m}$ and a maximum error of $7.05 \mathrm{~m}$ with $95 \%$ probability, versus the $3.93 m$ and $8.86 m$ of Current-RSS-DE, respectively.

Regarding the real scenario, the results are analogous as we can see in Figure 4. Current+PastRSS-DE provides better accuracies in the estimations in all percentiles, and the differences in terms of distance error increases for higher percentiles. In this case, the differences in accuracy for the percentiles 25, 50, 75 and 95 are $0.51 \mathrm{~m}, 0.90 \mathrm{~m}, 1.45 \mathrm{~m}$ and $1.35 \mathrm{~m}$, respectively. The results also confirm the bigger robustness of this approach. Its average (median) distance error is $4.4 \mathrm{~m}$, versus $5.3 \mathrm{~m}$ for Current-RSS-DE, whereas the 95 percentile is $10.5 \mathrm{~m}$, versus the $11.8 \mathrm{~m}$.

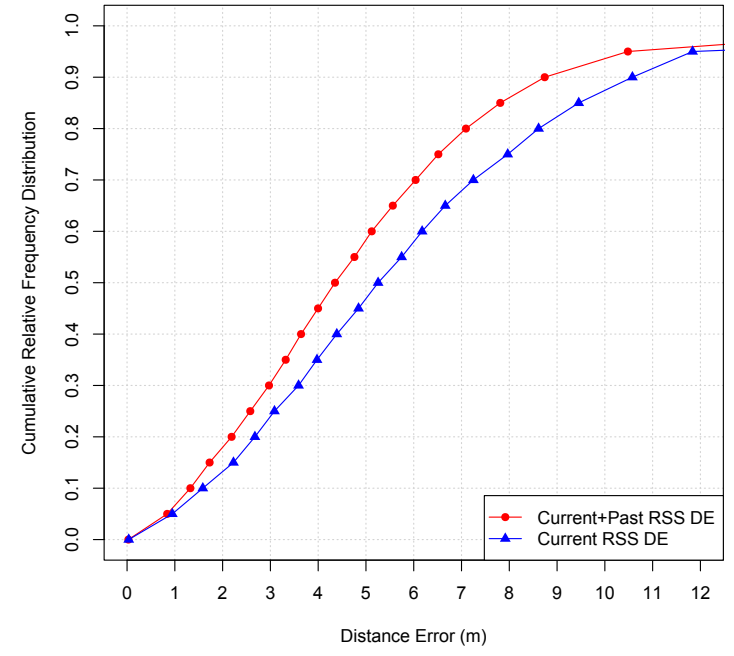

Figure 4: Cumulative Relative Frequency Distribution (CRFD) for the distance errors (in meters) of the estimations of Current-RRS-DE (blue-triangle line) and Current+Past-RSS-DE (red-circle line) in the real scenario.

\subsection{Comparison versus reference algorithms}

This section aims at comparing the performance of the approach presented in this paper (Current+Past-RSS-DE) versus similar methods proposed in the literature, in order to assess whether its results are competitive or not. To this end, we took the four methods proposed in [16] and [10]. We chose them because of the following reasons: they are methods that provide competitive results in terms of accuracy in the location estimates; their approaches are also based on the path-loss model; they use the same heuristic rules described in Section 2 ; and they have been tested in the same scenarios described in Section 4 and using the same RSS measurements, which makes the comparison easier and fairer. We provide a brief description of the four methods below (the interested read may refer to the former references for more details):

- LSM-RSS- $\Lambda$ : This method estimates the location of the MS using only RSS measurements. Its working is divided in two phases. First, the algorithm estimates the path-loss factors of the propagation models solving a equation system that relates these factors with the RSS measurements and using the same heuristics constraints shown in Section 2 to limit the space of feasible solutions. The path-loss factors in turn allow obtaining estimations of the distances from the MS to the APs which are then use, in the second phase, to estimate the position of the MS by a Least-Square Multilateration (LSM) method.

- LSM-RTT: This method uses a time-based measure, called Round-Trip Time (RTT), to estimate the location of the MS. This approach is 


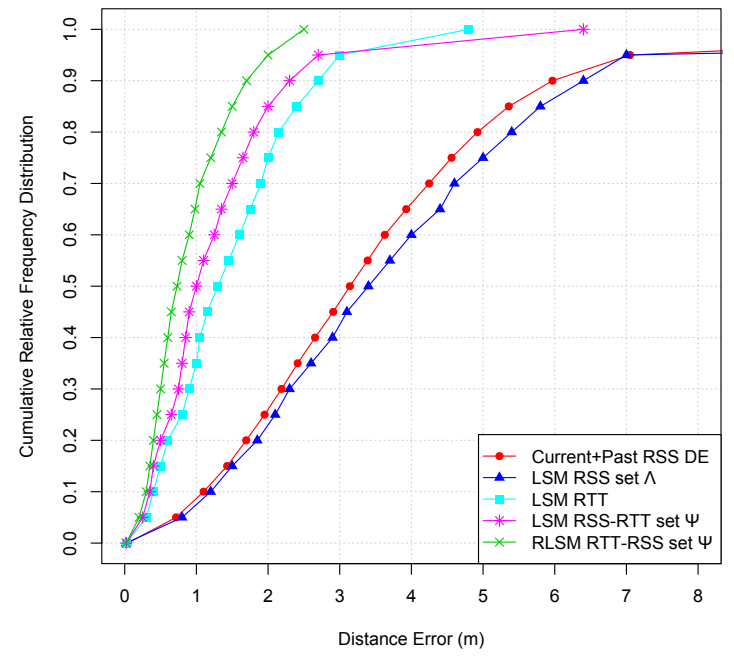

Figure 5: Cumulative Relative Frequency Distribution (CRFD) for the distance errors (in meters) of the location estimates for Current+PastRSS-DE and the reference algorithms LSM-RSS- $\Lambda$, LSM-RTT, LSM-RSS-RTT- $\Psi$, RLSM-RSS-RTT- $\Psi$, in the simulated scenario.

similar to the last one but using the RTT metric. First, it calculates the distances to APs from the RTT measurements and then, it also estimates the MS position using LSM.

- LSM-RSS-RTT- $\Psi$ : It is an hybrid of the two former methods that uses simultaneously RSS and RTT measurements to estimate distances, and LSM to calculate the position.

- RLSM-RSS-RTT- $\Psi$ : It is a variation of LSM-RSS-RTT- $\Psi$. The distances are computed in the same way, but the MS location is estimated by another method called Robust Least-Square Multilateration (RLSM).

It is important to note that LSM-RSS- $\Lambda$ is the only method that bases its estimations just on RSS measurements. This means that the comparison versus our proposal is only fair with respect to this method given that time-based measurements, as RTT, are better correlated with position than RSS measurements [20]. The main problem of timebased metrics is its low availability in common and inexpensive wireless systems unlike RSS indicators. Despite of this fact, we decided to include the RTTbased methods in order to check the competitiveness of our method versus high-performing indoor location systems. The parameter settings of these methods were the same used in [16] and [10].

Figures 5 and 6 display the CRDF for the distance errors obtained by the four reference methods and our proposal (Current+Past RSS DE) for the simulated and the real scenario, respectively. Looking at Figure 5, Current+Past-RSS-DE improves LSM-RSS- $\Lambda$ in virtually all percentiles of the distribution except for the 95, where they show a similar accuracy. Regarding the RTT-based methods, the performance of our proposal is clearly worse for

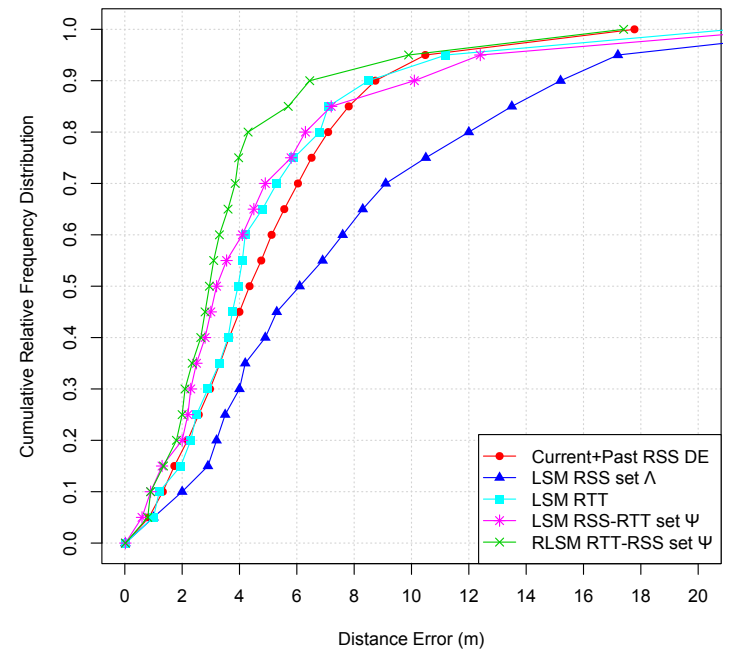

Figure 6: Cumulative Relative Frequency Distribution (CRFD) for the distance errors (in meters) of the location estimates for Current+PastRSS-DE and the reference algorithms LSM-RSS- $\Lambda$, LSM-RTT, LSM-RSS-RTT- $\Psi$, RLSM-RSS-RTT- $\Psi$, in the real scenario.

this scenario. However, when we consider the real scenario, where the RTT measurements are subject to a higher noise, these differences are much lower. In this case, our proposal outperforms LSM-RSS- $\Lambda$ and obtains distance errors similar to those obtained by RTT-based methods for higher percentiles. Although LSM-RTT, LSM-RSS-RTT- $\Psi$ and RLSMRSS-RTT- $\Psi$ show a better average (median) performance, the maximum error at a confidence level of 0.95 provided by our method is similar or better than that provided by these three algorithms, which proves the robustness of our proposal.

\section{Conclusions}

In this paper we have presented a new optimization approach for indoor location, based on RSS metrics, that unlike common optimization approaches for this purpose, it uses current and past measurements to estimate the MS location assuming unknown path-loss exponents. To deal with the bigger complexity of the underlying optimization problem, we developed a DE algorithm, given the good results of these methods in continuous multimodal search spaces, a feature present in the problem addressed here.

To validate our proposal, we used two scenarios, one simulated and one real, where a MS followed a trajectory that simulated to a person walking. The experimentation done aimed, on the one hand, at analyzing the benefits of estimating locations from current and previous RSS measurements versus using only the current ones, and on the other hand, at comparing the competitiveness of our proposal versus four high-performing methods in literature.

After analyzing the results we can draw various 
conclusions: 1) using both current and past RSS measurements leads to more accurate and more robust estimations; 2) our proposal outperforms the reference method that bases its estimations just in RSS metrics, both in the simulated and the real scenario; and 3), the results of our proposal are similar, in terms of robustness, when compared versus the reference approaches that use the RTT metric.

To sum up, in our opinion, these results show, first, that by incorporating more information to the objective function we can improve the accuracy of optimization-based approaches for indoor location, and second, the bigger complexity of the resulting models can be successfully addressed by means of evolutionary algorithms.

\section{Acknowledgements}

This work has been supported by the research projects TEC2013-45585-C2-2-R from the Spanish Ministry of Economy and Competitiveness and PC2013-71A from the Basque Government.

\section{References}

[1] F. Gustafsson and F. Gunnarsson. Mobile positioning using wireless networks: possibilities and fundamental limitations based on available wireless network measurements. IEEE Signal Processing Magazine, 22(4):41-53, 2005.

[2] M. B. Kjærgaard. A taxonomy for radio location fingerprinting. In Location- and ContextAwareness, pages 139-156. Springer, 2007.

[3] T. Roos, P. Myllymäki, H. Tirri, P. Misikangas, and J. Sievänen. A probabilistic approach to WLAN user location estimation. International Journal of Wireless Information Networks, 9(3):155-164, 2002.

[4] A. Ramachandran and S. Jagannathan. Spatial diversity in signal strength based WLAN location determination systems. In 32nd IEEE Conference on Local Computer Networks ( LCN 2007), 2007.

[5] L. F. M. de Moraes and B. A. A. Nunes. Calibration-free WLAN location system based on dynamic mapping of signal strength. In Proceedings of the international workshop on Mobility management and wireless access - MobiWac'06, 2006.

[6] F. Seco, A.R. Jimenez, C. Prieto, J. Roa, and K. Koutsou. A survey of mathematical methods for indoor localization. In IEEE International Symposium on Intelligent Signal Processing (WISP 2009), pages 9-14, 2009.

[7] Y. Qi. Wireless geolocation in a non-line-ofsight environment. PhD thesis, Princeton University, 2003.

[8] N. Patwari, A.O. Hero, M. Perkins, N.S. Correal, and R.J. OD́ea. Relative location estimation in wireless sensor networks. IEEE Tran- sanctions on Signal Processing, 51(8):21372148, 2003.

[9] A. Narzullaev, Y. Park, and H. Jung. Accurate signal strength prediction based positioning for indoor WLAN systems. In 2008 IEEE/ION Position, Location and Navigation Symposium. IEEE, 2008.

[10] A. Bahillo, S. Mazuelas, R. M. Lorenzo, P. Fernández, J. Prieto, R. J. Durán, and E. J. Abril. Accurate and integrated localization system for indoor environments based on IEEE 802.11 round-trip time measurements. EURASIP Journal on Wireless Communications and Networking, 2010:1-13, 2010.

[11] T. Bartz-Beielstein, J. Branke, J. Mehnen, and O. Mersmann. Evolutionary algorithms. WIREs Data Mining and Knowledge Discovery, 4(3):178-195, 2014.

[12] R. Chiong, T. Weise, and Z. Michalewicz. Variants of evolutionary algorithms for real-world applications. Springer, 2012.

[13] S. Das and P. N. Suganthan. Differential evolution: A survey of the state-of-the-art. IEEE Transactions on Evolutionary Computation, 15(1):4-31, 2011.

[14] K. Pahlavan and A. H. Levesque. Wireless information networks, volume 95. John Wiley \& Sons, 1995.

[15] S.D. Chitte, S. Dasgupta, and Zhi Ding. Distance estimation from received signal strength under log-normal shadowing: Bias and variance. IEEE Signal Processing Letters, 16(3):216-218, 2009.

[16] S. Mazuelas, A. Bahillo, R. M. Lorenzo, P. Fernandez, F. A. Lago, E. Garcia, J. Blas, and E. J. Abril. Robust indoor positioning provided by real-time RSSI values in unmodified WLAN networks. IEEE Journal of Selected Topics in Signal Processing, 3(5):821-831, 2009.

[17] P. Krishnan, A.S. Krishnakumar, Wen-Hua Ju, C. Mallows, and S.N. Gamt. A system for LEASE: location estimation assisted by stationary emitters for indoor RF wireless networks. In Proceedings of the Joint Conference of the IEEE Computer and Communications Societies (INFOCOM'04), volume 2, pages 1001-1011, 2004.

[18] X. Li. RSS-based location estimation with unknown pathloss model. IEEE Transactions on Wireless Communications, 5(12):3626-3633, 2006.

[19] R. Storn and K. Price. Differential evolution - a simple and efficient heuristic for global optimization over continuous spaces. Journal of Global Optimization, 11(4):341-359, 1997.

[20] D. Dardari, A. Conti, U. Ferner, A. Giorgetti, and M.Z. Win. Ranging with ultrawide bandwidth signals in multipath environments. Proceedings of the IEEE, 97(2):404-426, 2009. 